\title{
Improved gut microbiota features after the resolution of SARS-CoV-2 infection
}

\author{
Flavio De Maio ${ }^{1,2}$, Gianluca laniro 3,4, Gaetano Coppola4, Francesco Santopaolo4 ${ }^{4}$, Valeria Abbate ${ }^{4}$, \\ Delia Mercedes Bianco ${ }^{1,2}$, Fabio Del Zompo ${ }^{4}$, Giuseppe De Matteis ${ }^{5}$, Massimo Leo ${ }^{4}$, Antonio Nesci, \\ Alberto Nicoletti ${ }^{4}$, Maurizio Pompili ${ }^{3,4}$, Giovanni Cammarota ${ }^{3,4}$, Brunella Posteraro ${ }^{6,2}$, Maurizio Sanguinetti ${ }^{1,2}$, \\ Antonio Gasbarrini ${ }^{3,4}$ and Francesca Romana Ponziani ${ }^{3,4,7^{*}}$ (D)
}

\begin{abstract}
Background: The severe acute respiratory syndrome coronavirus 2 (SARS-CoV-2) has a tropism for the gastrointestinal tract and several studies have shown an alteration of the gut microbiota in hospitalized infected patients. However, long-term data on microbiota changes after recovery are lacking.

Methods: We enrolled 30 patients hospitalized for SARS-CoV-2-related pneumonia. Their gut microbiota was analyzed within $48 \mathrm{~h}$ from the admission and compared with (1) that of other patients admitted for suspected bacterial pneumonia (control group) (2) that obtained from the same subject 6 months after nasopharyngeal swab negativization.

Results: Gut microbiota alpha-diversity increased 6 months after the resolution of SARS-CoV-2 infection. Bacteroidetes relative abundance was higher $(\approx 36.8 \%)$ in patients with SARS-CoV-2, and declined to $18.7 \%$ when SARS-CoV-2 infection resolved $(p=0.004)$. Conversely, Firmicutes were prevalent $(\approx 75 \%)$ in controls and in samples collected after SARS-CoV-2 infection resolution ( $p=0.001$ ). Ruminococcaceae, Lachnospiraceae and Blautia increased after SARS-CoV-2 infection resolution, rebalancing the gut microbiota composition.
\end{abstract}

Conclusion: SARS-CoV-2 infection is associated with changes in the gut microbiome, which tend to be reversed in long-term period.

Keywords: Gut microbiota, SARS-CoV-2, COVID-19, Pneumonia

\section{Background}

Several studies to date have analyzed the gut microbiota of patients with severe acute respiratory syndrome coronavirus 2 (SARS-CoV-2) infection both during and after disease resolution [1, 2]. However, information on long-term follow-up is lacking. Therefore, we evaluated changes to the gut microbiome six months after SARS-CoV-2 infection resolution in 30 Italian patients

\footnotetext{
${ }^{*}$ Correspondence: francesca.ponziani@gmail.com

${ }^{7}$ Division of Internal Medicine and Gastroenterology, Hepatology Unit, Fondazione Policlinico Universitario Agostino Gemelli IRCCS, Università Cattolica del Sacro Cuore, Rome, Italy

Full list of author information is available at the end of the article
}

hospitalized for pneumonia in our center during the first wave of the pandemic.

\section{Study design and results}

Faecal samples of 31 SARS-CoV-2-positive patients were harvested within $48 \mathrm{~h}$ from admission and prior to transfer to the intensive care unit (ICU), in order to minimize any impact of the pharmaceutical treatment on the gut microbiome, and again six months after discharge. During this , patients were regularly interviewed and none of them reported any infection/antibiotic treatment or symptoms of new onset. Eighteen patients hospitalized in the same period for SARS-CoV-2-unerlated original author(s) and the source, provide a link to the Creative Commons licence, and indicate if changes were made. The images or other third party material in this article are included in the article's Creative Commons licence, unless indicated otherwise in a credit line to the material. If material is not included in the article's Creative Commons licence and your intended use is not permitted by statutory regulation or exceeds the permitted use, you will need to obtain permission directly from the copyright holder. To view a copy of this licence, visit http://creativecommons.org/licenses/by/4.0/. The Creative Commons Public Domain Dedication waiver (http://creativeco mmons.org/publicdomain/zero/1.0/) applies to the data made available in this article, unless otherwise stated in a credit line to the data. 
pneumonia served as control group. Following hospital admission, at the time of fecal specimen collection, both control group (94.7\%) and patients showing SARS-CoV-2 related pneumonia $(71 \%)$ were receiving the same antibiotic treatment schedule, as per Hospital protocol. Faecal samples analysis was conducted as described in the Additional file 4. Characteristics of the study population are shown in Table 1. Gut microbiota alpha-diversity was similar between patients affected by either SARS$\mathrm{CoV}-2$ or non-SARS-CoV-2 pneumonia; it showed a slight enhanced trend after SARS-CoV-2 negativization, increasing significantly after the resolution of SARS$\mathrm{CoV}-2$ infection when compared with the control group $(\mathrm{p}=0.346, \mathrm{p}=0.043$ and $\mathrm{p}=0.048$, Kruskal-Wallis Test, for Shannon index, inverse Simpson index and Pielou's Evenness, respectively). In particular, equitability among bacterial species increased and appeared to be driven by microbial changes ensuing SARS-CoV-2 infection resolution (Fig. 1A). The PCoA of inter-individual variation based on weighted UniFrac distance showed a slender split among the study groups, although it was not statistically significant $(\mathrm{p}=0.114$, PERMANOVA; Additional file 1: Figure S1). As shown in Fig. 1B, Bacteroidetes relative abundance was higher $(\approx 36.8 \%)$ in SARS-CoV-2 positive patients than in those with SARSCoV-2 negative pneumonia $(\approx 13.6 \%)$, and declined to $18.7 \%$ when SARS-CoV-2 infection resolved $(\mathrm{p}=0.004$,
Kruskal-Wallis Test). Conversely, Firmicutes were prevalent $(\approx 75 \%)$ in controls and in samples collected after SARS-CoV-2 infection resolution, while the relative abundance was lower $(\approx 50 \%)$ in SARS-CoV-2 positive pneumonia group ( $\mathrm{p}=0.001$, Kruskal-Wallis Test).

Focusing on the twenty most represented bacterial genera, we observed specific patterns of Bacteroides, Blautia and Enterococcus variations among groups (Fig. 1C). Particularly, Bacteroides and Enterococcus genera appeared to correlate inversely, with the former genus being increased in SARS-CoV-2 positive patients compared to the other groups $(\mathrm{p}=0.003)$, whereas the latter genus showed a decreasing trend $(\mathrm{p}=0.082)$. Finally, Blautia increased after SARS-CoV-2 infection resolution, rebalancing the gut microbiota composition $(\mathrm{p}=0.029)$. As expected, linear discriminant analysis (LDA) showed that bacterial elements belonging to Bacteroidetes (i. e., Oscillibacter, Ruminococcaceae DTU089, Bacteroidaceae, Bacteroidia, Parabacteroides and Tannerellaceae) were enriched in SARS-CoV-2 positive patients, whereas Lactobacillales, Streptococcus, Staphylococcus and Acidoaminococcus were increased in those with SARS-CoV2-unrelated pneumonia ( $\mathrm{p}<0.05$, Kruskal-Wallis Test; Fig. 1D). Conversely, Lachnospiraceae (i. e., NK4A136 group, Fusicantibacter and Roseburia) and Ruminococcaceae UCG-013 were increased after SARS-CoV-2 infection resolution. DESeq2 analysis reported substantially

Table 1 Clinical and demographic features of patients included in the study

\begin{tabular}{|c|c|c|c|}
\hline & SARS-CoV2 + (31) & SARS-CoV2 - (18) & $\mathrm{p}$ value \\
\hline Age & $66.7 \pm 14.4$ & $67.1 \pm 17.5$ & 0.372 \\
\hline \multicolumn{4}{|l|}{ Sex } \\
\hline Males & $23(74.2 \%)$ & $11(61.1 \%)$ & 0.357 \\
\hline Females & $8(25.8 \%)$ & $7(38.9 \%)$ & \\
\hline Gastrointestinal symptoms & $13(41.9 \%)$ & $5(27.8 \%)$ & 0.249 \\
\hline Respiratory failure* & $22(73.3 \%)$ & $6(33.3 \%)$ & 0.012 \\
\hline \multicolumn{4}{|l|}{ ARDS categories ${ }^{\mathrm{a}}$} \\
\hline III & $14(63.6 \%)$ & $5(83.3 \%)$ & \\
\hline$\|$ & $7(31.8 \%)$ & $1(16.7 \%)$ & \\
\hline । & $1(4.5 \%)$ & 0 & \\
\hline History of chronic lung disease & $4(12.9 \%)$ & $3(16.7 \%)$ & 0.512 \\
\hline \multicolumn{4}{|l|}{ Treatment during hospitalization } \\
\hline Antibiotics & $22(71 \%)$ & $18(94.7 \%)$ & 0.05 \\
\hline Antivirals & $27(90 \%)$ & $3(16.7 \%)$ & 0.00 \\
\hline Hydroxychloroquine & $29(93.5 \%)$ & $2(10.5 \%)$ & 0.017 \\
\hline Anti-IL-6 receptor monoclonal antibodies & $8(25.8 \%)$ & 0 & \\
\hline
\end{tabular}

Numeric variables are reported as mean \pm standard deviation, categorical ones as frequencies and percentages

*Number of patients with respiratory failure during hospitalization $\left(\mathrm{PaO}_{2} / \mathrm{FiO}_{2}\right.$ ratio $\left.<300\right)$

Significance was evaluated by Wilcoxon signed rank test and chi-square test (IBM SPSS Statistics)

SARS-CoV-2 severe acute respiratory syndrome coronavirus 2; IL interleukin

a III $\mathrm{PaO}_{2} / \mathrm{FiO}_{2}$ ratio 200-300, II PaO2/FiO2 ratio 100-200, I PaO2/FiO2 ratio $<100$ 

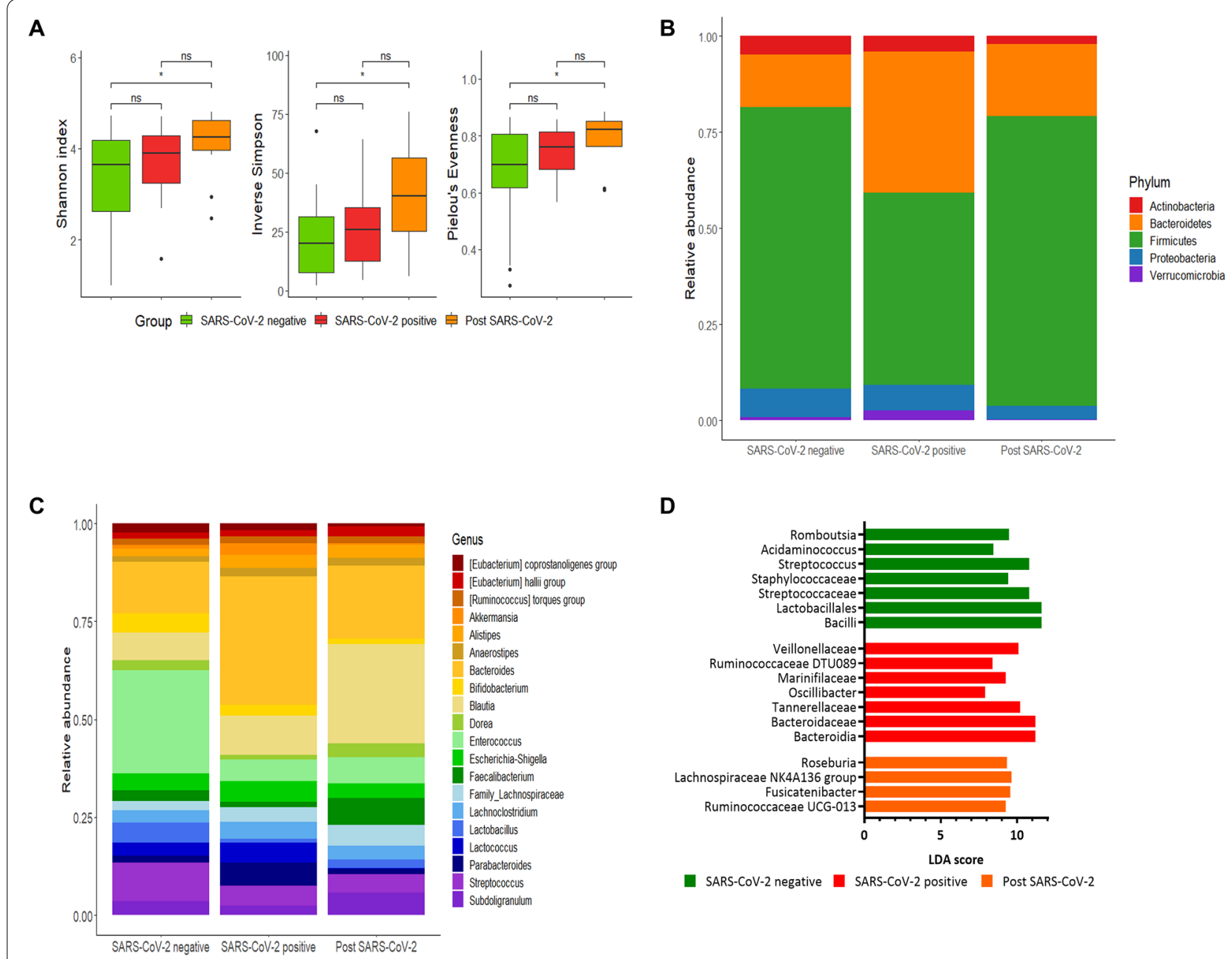

D

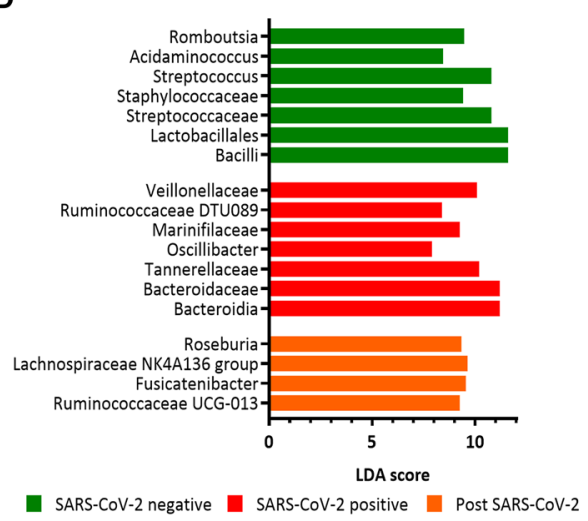

Fig. 1 Gut microbiota analysis of patients with or without severe acute respiratory syndrome coronavirus 2 (SARS-CoV-2) infection. A alpha diversity measures; B, C phyla and genera distribution; D linear discriminant analysis (LDA) effect size (LEfSe) highlighting differently abundant taxa between the study groups

the same results (Additional file 2: Table S1, Additional file 3: Table S2).

\section{Discussion}

Our study conducted in a Western population confirms that gut microbiota alpha diversity is similar in patients with SARS-CoV-2 related or unrelated pneumonia. Nevertheless, we observed an increase in alpha-diversity after the resolution of SARS-CoV-2 acute infection. This may be related to the effects of SARS-CoV-2 on the gut microbiota, but the contribution of drugs (e.g., antibiotics) administered before hospitalization or during its initial phase may not be negligible. Nevertheless, alpha-diversity modification may be not sufficient to assess recovery of the healthy status.
Previous Chinese studies [3-5] compared the gut microbiota of patients with SARS-CoV-2-related and community-acquired pneumonia, confirming a surge of opportunistic pathogens and a reduction in commensals elements. Our data confirm Bacteroidetes enrichment in patients with COVID-19 [3]. Conversely, Firmicutes appeared to decline, whereas no significant variation was observed for Actinobacteria [3]. During SARS-CoV-2 infection, inflammation is a key determinant of disease severity [6-8]. Interestingly, cytokine storm is positively associated with Bacteroides relative abundance [3]. Conversely, Blautia with its anti-inflammatory properties may play an important role in the recovery from COVID-19 [9]. Indeed, interleukin-10 (IL-10) serum levels significantly decrease with the reduction of Ruminococcus obeum (otherwise Blautia) 
abundance [3], and this correlates with the failure in controlling host's inflammatory response. This study suffers of the limitations of the small sample size, but our results corroborate those achieved on populations with different ethnicity. Even though many factors can affect the gut microbiota, our findings support the hypothesis of a specific impact of SARS-CoV-2 infection on the gut microbiota.

\section{Conclusions}

This study supports the previous evidence that SARSCoV-2 infection is associated with changes in the gut microbiome. However, many gut microbiome-related factors could influence the course of COVID-19, calling for more studies in the next future.

\section{Supplementary Information}

The online version contains supplementary material available at https://doi. org/10.1186/s13099-021-00459-9.

Additional file 1. Principal coordinate analysis (PCOA) on weighted UniFrac distance of the gut microbiome of patient with severe acute respiratory syndrome coronavirus 2 (SARS-CoV-2)-related pneumonia, who were tested before and after resolution of the infection, and patients with SARS-CoV-2 unrelated pneumonia.

Additional file 2. DESeq2 analysis.

Additional file 3. DESeq2 analysis.

Additional file 4. Supplementary methods.

\section{Acknowledgements}

None.

\section{Authors' contributions}

FDM analyzed samples, interpreted data, designed the study, wrote and approved the final version of the paper; FRP interpreted data, designed the study, wrote and approved the final version of the paper; DMB analyzed and interpreted data; GI, FS, VA, GDM, AN, MP, GC, BP, MS, AG interpreted data, approved the final version of the paper; GC, FDZ, ML, AN collected samples, interpreted data, approved the final version of the paper. All authors read and approved the final manuscript.

\section{Funding}

None.

\section{Availability of data and materials}

Available upon reasonable request.

\section{Declarations}

Ethics approval and consent to participate

The local ethics committee of the Fondazione Policlinico Universitario Agostino Gemelli IRCCS approved the study (ID 3042).
Consent for publication

Not applicable.

\section{Competing interests}

The authors declare that they have no competing interests.

\section{Author details}

'Dipartimento di Scienze di Laboratorio e Infettivologiche, Fondazione Policlinico Universitario Agostino Gemelli IRCCS, Rome, Italy. ${ }^{2}$ Dipartimento di Scienze Biotecnologiche di Base, Cliniche Intensivologiche e Perioperatorie, Università Cattolica del Sacro Cuore, Rome, Italy. ${ }^{3}$ Dipartimento di Medicina e Chirurgia Traslazionale, Università Cattolica del Sacro Cuore, Rome, Italy. ${ }^{4}$ CEMAD Digestive Disease Center, Fondazione Policlinico Universitario Agostino Gemelli IRCCS, Rome, Italy. Internal Medicine, Fondazione Policlinico Universitario Agostino Gemelli IRCCS, Rome, Italy. ${ }^{6}$ Dipartimento di Scienze Mediche e Chirurgiche, Fondazione Policlinico Universitario A. Gemelli IRCCS, Rome, Italy. ${ }^{7}$ Division of Internal Medicine and Gastroenterology, Hepatology Unit, Fondazione Policlinico Universitario Agostino Gemelli IRCCS, Università Cattolica del Sacro Cuore, Rome, Italy.

Received: 27 July 2021 Accepted: 6 October 2021

Published online: 16 October 2021

\section{References}

1. Chhibber-Goel J, Gopinathan S, Sharma A. Interplay between severities of COVID-19 and the gut microbiome: implications of bacterial co-infections? Gut Pathog. 2021;13:14.

2. Yamamoto S, Saito M, Tamura A, Prawisuda D, Mizutani T, Yotsuyanagi H. The human microbiome and COVID-19: a systematic review. PLoS ONE. 2021. https://doi.org/10.1371/journal.pone.0253293.

3. Yeoh KY, Zuo T, Lui GCY, Zhang F, Liu Q, Li AY, et al. Gut microbiota composition reflects disease severity and dysfunctional immune responses in patients with COVID-19. Gut. 2021;70:698-706.

4. Zuo T, Zhang F, Lui GCY, Yeoh YK, Li AYL, Zhan H, et al. Alterations in gut microbiota of patients with COVID-19 during time of hospitalization. Gastroenterology. 2020;159(3):944-955.e8.

5. Gu S, Chen Y, Wu Z, Chen Y, Gao H, Lv L, et al. Alterations of the gut microbiota in patients with coronavirus disease 2019 or H1N1 influenza. Clin Infect Dis. 2020;71:2669-78.

6. Ponziani FR, Del Zompo F, Nesci A, Santopaolo F, laniro G, Pompili M, et al. Liver involvement is not associated with mortality: results from a large cohort of SARS-CoV-2-positive patients. Aliment Pharmacol Ther. 2020;52:1060-8.

7. Ponziani FR, Nesci A, Del Zompo F, Santopaolo F, Pompili M, Gasbarrini A, et al. Correlation between liver function tests abnormalities and interleukin-6 serum levels in patients with SARS-CoV-2 infection. Gastroenterology. 2021;160:1891-3.

8. Del Zompo F, De Siena M, laniro G, Gasbarrini A, Pompili M, Ponziani FR. Prevalence of liver injury and correlation with clinical outcomes in patients with COVID-19: systematic review with meta-analysis. Eur Rev Med Pharmacol Sci. 2020;24:13072-88.

9. Liu X, Mao B, Gu J, Wu J, Cui S, Wang G, et al. Blautia-a new functional genus with potential probiotic properties? Gut Microbes. 2021;13:1-21.

\section{Publisher's Note}

Springer Nature remains neutral with regard to jurisdictional claims in published maps and institutional affiliations. 